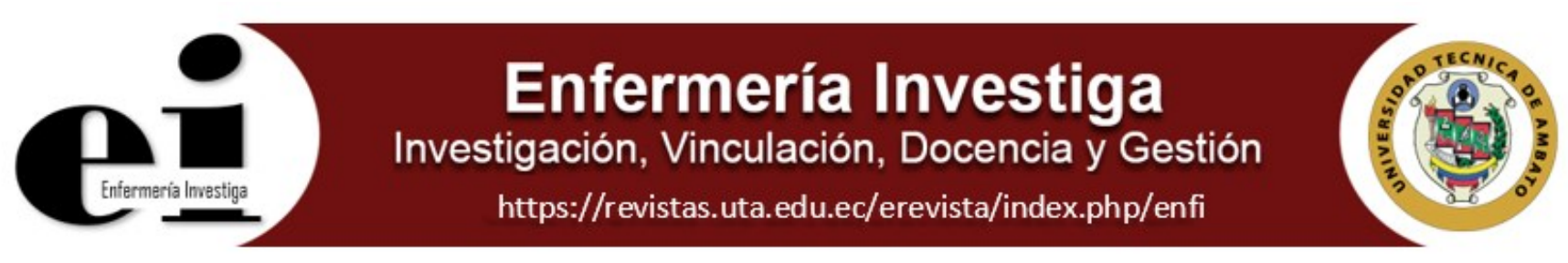

Artículo original

\title{
Medidas de bioseguridad en los padres de familia o tutor de un Hospital Pediátrico del Ecuador
}

Biosecurity measures in the parents or guardian of a Pediatric Hospital of Ecuador

Kiara Nataly Saca Valarezo ${ }^{1}$ https://orcid.org/0000-0003-2784-971X, Grace Ivanova Alvarado Guarderas ${ }^{1}$ https://orcid.org/0000-0001-8666-3531, Marlene Johana Chamba Tandazo ${ }^{1}$ https://orcid.org/0000-0001-6687-4569

${ }^{1}$ Universidad Técnica de Machala-Facultad de Ciencias Químicas y de la Salud- Carrera de Enfermería-Machala-Ecuador

Saca, K; Alvarado, G; Chamba, M. Medidas de bioseguridad en los padres de familia o tutor de un Hospital Pediátrico del Ecuador. Enferm Inv. 2020;5(3):32-38

2477-9172 / 2550-6692 Derechos Reservados (C 2020 Universidad Técnica de Ambato, Carrera de Enfermería. Este es un artículo de acceso abierto distribuido bajo los términos de la Licencia Creative Commons, que permite uso ilimitado, distribución y reproducción en cualquier medio, siempre que la obra original es debidamente citada.

Historia:

Recibido: 01 abril 2020

Aceptado: 01 junio 2020

Palabras Claves:

conocimiento, padres de familia

o tutor, bioseguridad.

Keywords: knowledge, parents or guardian, biosecurity

\begin{abstract}
Resumen
Introducción: las normas de bioseguridad actúan como un factor protector de infecciones intrahospitalarias al contrarrestar la exposición de riesgos biológicos producto de agentes infecciosos que se encuentran presentes en los centros de salud.

Objetivo: determinar los conocimientos de los padres de familia o tutor de las medidas de bioseguridad en el área de salud pediátrica Métodos: investigación no experimental, descriptivo, transversal, dirigido a 60 padres de familia o tutor que acompañaron a los pacientes hospitalizados en el área de pediatría.

Resultados: Se evidenció que los padres de familia o tutores tienen un $88,3 \%$ de conocimientos inadecuados sobre las medidas de bioseguridad en el área de pediatría, el $90 \%$ de los profesionales de salud y de enfermería del área de pediatría no realizan un proceso educativo de las normas de bioseguridad a los padres, $58.3 \%$ de los padres en pocas ocasiones se lavan de las manos durante la hospitalización de sus hijos, $66 \%$ de los padres no utilizan el gel antiséptico, el $83.3 \%$ de los padres muy pocas veces se lavan las manos luego de salir del área de pediatría, el $73.3 \%$ padres no clasifica los desechos en su respectivo depósito de basura.

Conclusiones: Los padres de familia o tutores tienen conocimientos inadecuados sobre las medidas de bioseguridad en el área de pediatría El personal de salud debe brindar información relevante sobre las medidas de bioseguridad para mejorar los hábitos higiénicos saludables para la prevención de las enfermedades intrahospitalarias.
\end{abstract}




\section{Introducción}

El Manual de normas de bioseguridad para la red de servicios de salud en el Ecuador se refiere a la bioseguridad como "Las normas de comportamiento y manejo preventivo, del personal de salud, frente a microorganismos potencialmente infecciosos, con el propósito de disminuir la probabilidad de adquirir infecciones en el medio laboral, haciendo énfasis en la prevención mediante la asepsia y el aislamiento"(1)

Este concepto con el paso del tiempo se ha ampliado, indicando que la bioseguridad es la aplicación de los conocimientos, técnicas y equipamientos para prevenir a personas, laboratorios, áreas hospitalarias y medio ambiente de la exposición a agentes potencialmente infecciosos o considerados de riesgo biológico, entendiéndose como riesgo biológico a la probabilidad de adquirir una enfermedad a partir del contacto con material biológico (2).

De acuerdo, con el autor Romero, las medidas de bioseguridad hospitalaria tiene como objetivo contrarrestar la exposición de riesgos biológicos producto de agentes infecciosos que se encuentran presentes en centros de salud, pacientes, visitas familiares, instrumentos médicos, entre otras fuentes de contaminación; por tanto, la aplicación de las medidas y normas de bioseguridad actuará como un factor protector ante la presencia de riesgos nocivos (3).

El Ministerio de Salud Pública, 2011 (MSP), creo un manual de bioseguridad cuyo propósito es el fortalecimiento, seguridad y atención de calidad en los servicios de salud, a través de la aplicación de medidas preventivas como: formas de esterilización, lavado de manos, clasificación de desechos hospitalarios, etc. Poco a poco se han ido perfeccionando y creando nuevas guías de bioseguridad como el Manual Bioseguridad para los establecimientos de salud publicado por el MSP en el 2016, el que tiene como objetivo principal estandarizar las medidas de bioseguridad en el país, para así disminuir el riesgo de infecciones asociados en el cuidado de la salud de los trabajadores y usuarios (4). Además existen cuatro principios que engloban la bioseguridad: el autocuidado, la universalidad, el uso de barreras y las medidas de eliminación (5).

El autocuidado se define como la capacidad de ejecutar acciones cotidianas que permitan protegernos de agentes biológicos que pueden dañar nuestra salud, la universalidad nace del peligro biológico que representa cada individuo, donde debemos considerar a todos como altamente portadores de microorganismos patógenos, el uso de barreras, consiste en equipos físicos que evitan la exposición directa de fluidos orgánicos altamente contaminantes, aquí se incluyen el uso de bata, guates y mascarilla, las medidas de eliminación de material contaminado comprenden las acciones necesarias para clasificar los materiales utilizados en la atención a pacientes y que deben ser clasificados y eliminados sin riesgo (5-7).
La Organización mundial de la Salud afirma que la higiene de manos es el método más eficaz para disminuir el traspaso de material infectante de un individuo a otro, ya sea por fricción con un preparado de base alcohólica o lavado con agua y jabón, con el objetivo de reducir o inhibir el crecimiento de microorganismos en las manos (6). El Lavado con agua y jabón antiséptico permite eliminar en forma significativa tanto la flora transitoria como la residente presente en las manos y la fricción antiséptica con un preparado de base de alcohol: con el objetivo de reducir flora residente y eliminar la flora transitoria presente en la piel Preparado de base alcohólica, etílico o isopropílico 63 al $70 \%(7,8)$

El lavado de manos debe realizarse teniendo en cuenta los 5 momentos del lavado de manos propuesto por la Organización Mundial de la Salud: antes del contacto con el paciente, antes de un procedimiento limpio/ aséptico, después del riesgo de exposición a fluidos corporales, después del contacto con el paciente y después del contacto con el entorno del paciente (9).

Dos de los cinco momentos para la higiene de las manos se producen antes del contacto con el paciente y los tres restantes se producen después del contacto o exposición a fluidos corporales, las indicaciones que corresponden a los momentos "antes" señalan la necesidad de prevenir el riesgo de transmisión microbiana al paciente y las indicaciones "después" pretenden prevenir el riesgo de transmisión microbiana al familiar o al área de asistencia (es decir, otros pacientes, sus inmediaciones y el entorno sanitario) (9).

El uso de las mascarillas en el área de pediatría es importante ya que existen salas donde hay pacientes con enfermedades respiratorias que pueden transmitirse tanto al personal de salud, como a los acompañantes, estas mascarillas pueden atrapar partículas grandes de fluidos corporales que contienen microrganismos infecciosos que son expulsados por una persona enferma $(10,11)$.

Otra de las actividades que los familiares están involucrados es la clasificación de los desechos que se generan dentro de la sala, estos pueden ser desechos comunes o generales que se colocan en tachos negros y los desechos infecciosos que se deben colocar en los tachos rojos, una tarea que es obviada y que al no ser ejecutada de manera correcta pone en riesgo al ambiente y comunidad. Además del factor económico que implica la eliminación de los mismos, por eso es importante una correcta clasificación al eliminarlos (12).

Los desechos comunes son aquellos que no representan un riesgo para la salud humana, ni para el medio ambiente por lo tanto no requieren de un tratamiento especial aquí se incluyen por ejemplo: plástico, papel, cartón y desechos de procedimientos médicos no contaminantes como yeso y vendas; Los desechos infecciosos en cambio son aquellos que contienen microrganismos patógenos y por tanto son considerados peligrosos para la salud y el medio 
ambiente, aquí se incluyen todo el material que ha estado en contacto con fluidos corporales como sangre, orina y heces $(13,14)$.

Sin embargo, es importante subrayar que no existe un manual o protocolo específico que abarque las medidas de bioseguridad correspondientes para los acompañantes del paciente durante su proceso de recuperación en las áreas hospitalarias donde es obligatorio contar con la presencia del padre de familia o tutor permanente, en el área pediatría que colaboran en la atención y cuidado del paciente pediátrico, se estima que aproximadamente un $25 \%$ de los niños menores de 18 años se ha hospitalizado al menos una vez en su vida, por lo tanto el índice de permanecer en un hospital es alto, además diversos estudios han determinado que existen un gran índice de reingresos hospitalarios en esta población, que pueden ser prevenidos si se tomaran en cuenta normas básicas de higiene y bioseguridad $(15,16)$.

Los riesgos producto de la utilización inadecuada de las medidas de bioseguridad generan un impacto negativo a pacientes, personal y profesionales de salud, considerando el grado de vulnerabilidad en el que se encuentran expuestos. De manera que, el conocimiento y la adecuada aplicación de estas normas evitarán que el índice de infecciones y contaminación se incremente en los sistemas de salud $(17,18)$, por estas razones el objetivo de esta investigación fue determinar los conocimientos de los padres de familia o tutor de las medidas de bioseguridad en el área de salud pediátrica.

\section{Materiales y métodos}

Se realizó un estudio no experimental, descriptivo, transversal, para evaluar el nivel de conocimiento de los padres de familia o tutor sobre las medidas de bioseguridad en el área de pediatría del hospital General Teófilo Dávila, la muestra fue de tipo censal porque se seleccionaron el total de 60 padres o tutores del área pediátrica del hospital.

Los datos fueron extraídos mediante un cuestionario de conocimientos de bioseguridad hospitalaria, la cual consta de las siguientes variables: proceso educativo y conocimientos de las normas de bioseguridad, lavado de las manos y utilización de mascarilla dentro del área de pediatría, dicho cuestionario consta de preguntas cerradas en escala Likert, con una duración total veinte minutos por aplicación. Consecuentemente se aplicó la técnica de observación con la finalidad de evidenciar si realizaban el lavado de manos de manera correcta o errónea en cada padre de familia, luego se empleó la evaluación post-charla cuya finalidad fue brindar información sobre la forma adecuada de utilizar las medidas de bioseguridad. los resultados fueron analizados mediante el programa estadístico SPSS versión 0.24

En las consideraciones éticas: esta investigación cumple con los principios de autonomía, beneficencia, no maleficencia, confidencialidad, con la aprobación del Comité de Ética, los padres de familia firmaron el consentimiento informado.

\section{Resultados}

Mediante el análisis del nivel de conocimientos de los padres de familia o tutores sobre las medidas de bioseguridad en el área de pediatría, se concluye que los resultados obtenidos muestran que el $88,3 \%$ es inadecuado, solo el $11.7 \%$ tienen conocimientos adecuado de las normas de bioseguridad

Tabla $\mathbf{N}^{\circ}$ 1. Nivel del conocimiento de los padres sobre las normas de bioseguridad

\begin{tabular}{ccc}
\hline $\begin{array}{l}\text { Nivel del } \\
\text { conocimiento de } \\
\text { los padres sobre } \\
\text { las normas de } \\
\text { bioseguridad }\end{array}$ & Frecuencia & Porcentaje \\
\hline Adecuado & 7 & \\
Inadecuado & 53 & 11,7 \\
\hline Total & 60 & 88,3 \\
\hline
\end{tabular}

Fuente: Hospital General Teófilo Dávila.

El proceso educativo del personal de enfermería a los padres en un $90 \%$ no suministran la información adecuada sobre las normas de bioseguridad como el lavado de manos, uso de mascarilla y/o clasificar los desechos y solo el $10 \%$ realizan proceso educativo adecuado

Tabla $N^{\circ} 2$. Proceso educativo del personal de enfermería sobre las normas de bioseguridad de los padres

\begin{tabular}{|c|c|c|}
\hline $\begin{array}{l}\text { Proceso } \\
\text { educativo del } \\
\text { personal de } \\
\text { enfermería } \\
\text { sobre las } \\
\text { normas de } \\
\text { bioseguridad de } \\
\text { los padres }\end{array}$ & Frecuencia & Porcentaje \\
\hline Adecuado & 10 & 10,0 \\
\hline Inadecuado & 90 & 90,0 \\
\hline Total & 60 & 100,0 \\
\hline
\end{tabular}

Fuente: Hospital General Teófilo Dávila.

La frecuencia del lavado de las manos de los padres un $41.7 \%$ realizan en forma constante, mientras que el $58.3 \%$ lo realiza en pocas ocasiones ya sea, porque no existen insumos necesarios para el lavado de manos o porque no saben sobre la importancia de la misma. 
Tabla N4. Utilización gel antiséptico para la desinfección de manos

\begin{tabular}{lcc}
\hline \multicolumn{3}{c}{ manos } \\
$\begin{array}{l}\text { Utilización } \\
\text { antiséptico } \\
\text { para la } \\
\text { desinfección } \\
\text { de manos }\end{array}$ & Frecuencia & Porcentaje \\
\hline Casi siempre & 7 & \\
Muy pocas veces & 13 & 11,6 \\
Nunca & 40 & 21,7 \\
\hline Total & 60 & 66,7 \\
\hline Fuente: Hospital General Teófilo Dávila. & 100,0 \\
\hline
\end{tabular}

Con respecto al lavado de las manos al salir del área de pediatría: El $83.3 \%$ de los padres muy pocas veces se lavan las manos luego de salir del área de pediatría y solo el $16.4 \%$ de los padres lo hacen casi siempre. manifestando verbalmente, que unos no se lavan las manos cuando ingresan, o después del contacto del familiar, existe una escasez grande sobre el lavado de manos, no saben sobre la importancia y que les ayuda a prevenir si lo hacemos en los momentos correctos.

Tabla $\mathbf{N}^{\circ}$ 5. Lavado de las manos al salir del área de pediatría

\begin{tabular}{|c|c|c|}
\hline $\begin{array}{l}\text { Lavado de } \\
\text { las manos } \\
\text { al salir del } \\
\text { área de } \\
\text { pediatría }\end{array}$ & Frecuencia & Porcentaje \\
\hline
\end{tabular}

\begin{tabular}{ccc} 
Casi siempre & 10 & 16,7 \\
Muy pocas veces & 50 & 83,3 \\
\hline Total & 60 & 100,0 \\
\hline
\end{tabular}

Fuente: Hospital General Teófilo Dávila.

En relación a la utilización de mascarilla dentro del área de pediatría: Un $3.3 \%$ de los padres utiliza mascarilla casi siempre, mientras que $13.2 \%$ muy pocas veces y el $83.5 \%$ nunca utiliza mascarilla dentro del área de pediatría, se evidencia la falta de información y capacitación por parte del personal de salud hacia los pacientes y padres de familia que se encuentran hospitalizados en esta área

Tabla $\mathbf{N}^{\circ} 6$. Lavado de las manos al salir del área de pediatría

\begin{tabular}{|c|c|c|}
\hline $\begin{array}{l}\text { Lavado de } \\
\text { las manos } \\
\text { al salir del } \\
\text { área de } \\
\text { pediatría }\end{array}$ & Frecuencia & Porcentaje \\
\hline
\end{tabular}

\section{Casi siempre}

Muy pocas veces
2

8
3,3

13,2

\begin{tabular}{ccc} 
Nunca & 50 & 83,5 \\
\hline Total & 60 & 100,0 \\
\hline
\end{tabular}

Fuente: Hospital General Teófilo Dávila.

En la clasificación de los desechos realizados por los padres en el área pediátrica el $73.3 \%$ no clasifica los desechos en su respectivo depósito de basura correspondiente, representando un porcentaje elevado de mala clasificación de desechos hospitalarios en los padres de familia que acompañan a los pacientes de pediatría y solo el $26.7 \%$ realiza la clasificación de los desechos en forma adecuada, porque no existen capacitaciones frecuentes sobre su gran importancia de la clasificación y las consecuencias que abarcan la clasificación de los desechos hospitalarios.

Tabla ํ⒎ Clasificación de los desechos por los padres Clasificación de los Frecuencia

Porcentaje desechos por los padres

\begin{tabular}{ccc} 
Muy pocas veces & 16 & 26,7 \\
Nunca & 44 & 73,3 \\
\hline Total & $\mathbf{6 0}$ & 100,0 \\
\hline Fuente: Hospital General Teófilo Dávila. &
\end{tabular}

\section{Discusión}

Diversos estudios concluyen que incluir a los padres de familia en el cuidado del niño hospitalizado, permite la pronta recuperación del mismo, en 1986 con el fin de proteger a los niños de los posibles peligros que pueden derivar de la situación de hospitalización e incluir a los padres dentro de los cuidados hospitalarios, se redactó una carta, por el parlamento Europeo donde se declararon algunos derechos del niño hospitalizado entre ellos: "Derecho del niño a estar acompañado de sus padres, o de la persona que los sustituya, el máximo tiempo posible, durante su permanencia en el hospital, no como espectadores pasivos sino como elementos activos de la vida hospitalaria, sin que ello comporte gastos adicionales; el ejercicio de este derecho no debe perjudicar en modo alguno ni obstaculizar la aplicación de los tratamientos a los que hay que someter al niño" $(19,20)$. En el Ecuador las áreas de internación pediátricas cumplen con este derecho por lo tanto se requiere un acompañante las 24 horas, debido a la interacción de tutores con el medio hospitalario es necesario que este contribuya al cuidado del menor, pero también que cuide su propia salud y la de los demás habitantes en su ámbito familiar.

Por lo tanto, los padres de familia o tutores deben conocer las normas de bioseguridad respectivas y aplicarlas sin embargo mediante en este estudio se pudo determinar que solo el $11,7 \%$ sabe que son las normas de bioseguridad y las aplican, el $83.3 \%$ por lo tanto esta población restante, tienen un alto riesgo de 
contaminarse o contraer una enfermedad nosocomial por el contacto directo con el paciente enfermo y su ambiente al desconocer cómo protegerse, ya que el $90 \%$ de ellos manifestó no haber recibido suficiente información por parte del personal de salud sobre temas de bioseguridad, el estudio de "Factores de riesgo asociados a infecciones por bacterias multirresistentes derivadas de la atención en salud en una institución hospitalaria de la ciudad de Medellín 2011-2014", confirma que entre los factores de asocian a las infecciones intrahospitalarias se encuentra el incumplimiento de las normas de bioseguridad $(21,22)$, debido a estas razones se necesitan programas educativos que incrementen las estrategias docentes de las enfermeras (23)

Durante la investigación se preguntó a los acompañantes si ellos consideraban que hospital era un lugar libre de gérmenes, el $100 \%$ de ellos respondieron que no, evidentemente se sabe que en los hospitales existe una gran cantidad de microorganismos que pueden contaminar a los pacientes o usuarios, sin embargo, no aplican las medidas básicas de bioseguridad, en un estudio realizado en España sobre "Microorganismos multirresistentes en los hospitales: un riesgo amenazante" concluyo que los hospitales son nichos ecológicos con elevado de riesgo de contaminar y causar infecciones por bacterias resistentes a los pacientes, personal de salud, y demás usuarios como los tutores (24).

En lo que respecta a la frecuencia del lavado de manos por parte de los acompañantes se determinó que 58\% se lava las manos muy pocas veces, y solo un $15 \%$ de ellos se lava las manos de manera adecuada y cumpliendo los 5 momentos del lavado de manos propuestos por la Organización mundial de la Salud, esto genera gran preocupación porque aumenta el riesgo de ocasionar una infección cruzada, un estudio realizado en Nicaragua sobre "Conocimientos, actitudes y prácticas relacionadas con las infecciones intrahospitalarias", concuerda al igual que varios estudios que "Existe una falta de conocimiento sobre las infecciones asociadas a la atención en salud, así como en el ejercicio de actitudes y prácticas correctas destinadas a su prevención y que es importante diseñar un programa de capacitación en este nivel de atención para tratar de disminuir las infecciones para todos los usuarios de los hospitales" $(24,25)$.

Un estudio sobre higienización de manos en un hospital en Perú, señala que el lavado de las manos puede ser usado como medida preventiva de diversas enfermedades y que incluso puede ser más efectiva que cualquier vacuna, es más hoy en día la falta de lavado de manos resultó una de las principales causas de morbilidad por gastroenteritis e infecciones respiratorias en niños, motivo por el cual es una actividad que debe ser reforzada por el personal de salud antes, durante, y después del egreso hospitalario (22-27)

En lo que respecta al uso de mascarillas el 83,5\% de acompañantes no las usan, a pesar de que dentro del área de pediatría existen salas de enfermedades respiratorias donde es indispensable su uso como protección personal, según el manual de Organización panamericana de la salud sobre infecciones respiratorias agudas en los niños, nos indica que las infecciones respiratorias agudas son una de las causas más comunes de mortalidad en los niños de los países en vías de desarrollo, usar las mascarillas por la tanto en estas áreas permite evitar diseminar estas afecciones a los demás niños hospitalizados y al tutor $(28,29)$.

En lo que respecta a la clasificación de los desechos hospitalarios, los pacientes indicaron en un 73,3 \% tener dificultad para clasificar los desechos, la guía de manejo de los desechos hospitalarios del Hospital de Instituto Ecuatoriano de Seguridad Social de Macas, indico que la mala clasificación de los desechos, genera riesgos que amenacen no solo la salud de la población hospitalaria, sino también contribuye al deterioro ambiental situación que afecta a todo la población, al verse expuestos al contacto con material infeccioso una vez que los desechos son trasladados fuera del hospital para su tratamiento o disposición final (30).

Lastimosamente no existen muchos estudios que nos permitan identificar el grado de implicación en la salud de los cuidadores en las diferentes áreas hospitalarias; y deja en evidencia que se requieren más investigaciones centradas en el punto de normas de bioseguridad para los acompañantes y poder confirmar estos resultados y además mejorar las intervenciones, que han avanzado durante los últimos años, información útil para el equipo de salud, mejorando lo que ya se ha implementado y para los acompañantes, conociendo cómo debe ser su participación y su actitud de bioseguridad durante la hospitalización (31)

\section{Conclusiones}

El nivel de conocimientos sobre las medidas de bioseguridad en los padres de familia o tutores de la institución investigada es deficiente, teniendo en cuenta que la aplicación de las normas de bioseguridad en los hospitales permiten disminuir la incidencia de contraer infecciones intrahospitalaria que son las responsables de considerable morbilidad, mortalidad, estancia hospitalaria y costos para las unidades de salud, no solo deben aplicarse por el personal de salud, si no por todas las personas que ingresan al medio hospitalario. El rol del Profesional de Enfermería debe estar enfocado a la educación activa y constante del familiar desde el ingreso hasta el egreso del paciente al que cuidan, brindar información relevante sobre el uso e importancia de estas medidas de bioseguridad ayudara a mejorar la salud, hábitos higiénicos-saludables para su benefició y prevengan enfermedades intrahospitalarias, solo así el tutor se convertirá en un coadyuvante fundamental en el proceso de recuperación del paciente sin exponer su propia salud y la del paciente al que cuida. 


\section{Conflicto de intereses}

Ninguno declarado por los autores.

\section{Financiación}

Autofinanciado.

\section{Agradecimientos}

Ninguno declarado por los autores.

\section{Referencias}

1. Bioseguridad, M. D. E. Subred Integrada De Servicios De Salud Sur E.S.E Manual De Bioseguridad Ea-Ma-02 V1. Manual De Bioseguridad Subred Integrada De Servicios De Salud Sur E.S.E EaMa-01. 2017; 1-87. Disponible en http://www.subredsur.gov.co/sites/default/files/planeacion/EA-MA-02 V1MANUAL DE BIOSEGURIDAD.pdf

2. Cantón, R., \& Morosini, M. I. Microorganismos multirresistentes en los hospitales: Un riesgo amenazante. Gastroenterologia y Hepatologia Continuad. 2010; 9(5):254-257. https://doi.org/10.1016/S1578-1550(10)70054-1

3. Castañeda-Narváez, J. L., \& Hernández-Orozco, H. G. Washing (hygiene) with soap and water. Acta Pediatrica de Mexico. 2016; 37(6): 355-357. https://doi.org/10.18233/APM37No6pp355-357

4. Castro Otazu, M., Coasaca Núñez, A., Benavente Vilca, L. Quenaya Bejarano, E., \& Cruz Soriano, J. Higienización del lavado de manos para disminuir infecciones hospitalarias dada la ejecución de un sistema de gestión de calidad en el Hospital Base III - Essalud Juliaca-Puno. Cátedra Villarreal. 2017; 5(1), 45-51. https://doi.org/10.24039/cv201751186

5. Chiong Lay, M., Leisewitz Velasco, A., Márquez Romegialli, F., Vironneau Janicek, L., Álvarez Santana, M., Tischler -Fundación Ciencia, N., \& Moreno Mauro, R. Manual de Normas de Bioseguridad y Riesgos Asociados Fondecyt-CONICYT. Chile. 2018. Disponible en https://www.conicyt.cl/fondecyt/files/2018/08/MANUAL-DE-NORMASDE-BIOSEGURIDAD.pdf

6. Concepción, E., Gracia, H., \& Montenegro, D. Manejo de Desechos Hospitalarios. Macas. 2016 Disponible en: http://dspace.unach.edu.ec/bitstream/51000/2538/2/UNACH-IPGENF-CLI-QUI-2016-ANX-003.1.pdf

7. De, F., Grado, E., Enfermería, E. N., Diez, L., Tutor, U., Luis, J., \& Caballero, V. Aspectos psicológicos del niño hospitalizado. Universidad de Valladolid 2016. Disponible en. http://uvadoc.uva.es/bitstream/handle/10324/24480/TFGH897.pdf? sequence=1\&isAllowed=y

8. $\quad$ Ecuador, M. de S. P. G. N. de la R. Control Y Mejoramiento De La Salud Pública Salud Ambiental Reglamento «Manejo De Los Desechos Infecciosos Para La Red De Servicios De Salud En E Ecuador». Control y Mejoramiento de la Sálud Pública Salud Ambiental. Quito 2010.Disponible en: http://simce.ambiente.gob.ec/sites/default/files/documentos/Jackson/ Control y mejoramiento de la salud pública - Salud Ambiental.pdf

9. Hernández Faure, C., González Treasure, A., González Rodríguez, I., \& de la Cruz Vázquez, R. Revista información científica. Revista Información Científica. 2019;98(1):17-28. Recuperado de http://scielo.sld.cu/scielo.php?pid=S1028-

99332019000100017\&script=sci_abstract\&tlng=pt

10. Londoño Restrepo, J., Macias Ospina, I. C., \& Ochoa Jaramillo, F. L. 2016; Factores de riesgo asociados a infecciones por bacterias multirresistentes derivadas de la atención en salud en una institución hospitalaria de la ciudad de Medellín 2011-2014. Infectio. 2016,20(2):77-83. https://doi.org/10.1016/j.infect.2015.09.002

11. López López, S. P. 2011; Riesgos biológicos del personal de enfermería relacionado con el manejo de bioseguridad en el área de quirófano del Hospital Provincial General Latacunga en el periodo diciembre 2013 junio 2014. (19). Disponible en: http://repo.uta.edu.ec/bitstream/handle/123456789/4389/Andrea Paola Simon Gordillo.pdf?sequence $=1$
12. Mamani, S. S. N. "Conocimiento sobre manejo de los residuos sólidos hospitalarios por el personal de salud del hospita Santa Rosa Puerto de Maldonado - 2016". Universidad Nacional Amazónica de Madre de Dios - UNAMAD. Universidad Nacional Amazónica de Madre de Dios. Disponible en: http://repositorio.unamad.edu.pe/handle/UNAMAD/205

13. Ministerio de Salud Pública del Ecuador. 2016; Bioseguridad para los establecimientos de salud. Manual. Ministerio de Salud Publica. Recuperado de www.salud.gob.ec

14. Ministerio de Salud Pública del Ecuador. Manual Seguridad del Paciente-Usuario. Registro Oficial. 2016, 80. Recuperado de http://www.calidadsalud.gob.ec/wpcontent/uploads/2017/08/MANUAL-DE-SEGURIDAD-DELPACIENTE.pdf

15. Organización Mundial de la Salud. Manual técnico de referencia para la higiene de las manos, SAVE LIVES Clean Your Hands $\quad \S \quad 2010 ; \quad$ Recuperado de https://apps.who.int/iris/bitstream/handle/10665/102537/WHO_IER_P SP_2009.02_spa.pdf?sequence $=1$

16. Organizacion Panamericana de la Salud. 1992; Infecciones respiratorias agudas en los niños: tratamiento de casos en hospitales pequeños. Recuperado http://iris.paho.org/xmlui/bitstream/handle/123456789/3111/Infeccione $\mathrm{s}$ respiratorias agudas en los ninios Tratamiento de casos en hospitales

pequenios.pdf;jsessionid=8B7332EB4A28D4CC1A487C3AD0682600 ?sequence $=1$

17. Para, T., El, O., \& De, T. s. f; Educación que brinda la enfermera al alta hospitalaria a padres de niños posoperados de cirugía abdominal en un Hospital Local Chiclayo, 2018. Universidad Católica Santo Toribio de Mogrovejo Facultad de Medicina Escuela de Enfermería.

18. Pérez-Moreno, J., Leal-Barceló, A. M., Márquez Isidro, E., Toledo-del Castillo, B., González-Martínez, F., González-Sánchez, M. I., \& Rodríguez-Fernández, R. 2019; Detection of risk factors for preventable paediatric hospital readmissions. Anales de Pediatria. 2019;91(6):365-370. https://doi.org/10.1016/j.anpedi.2018.12.003

19. Quimbiulco Lanchimba, L. D., \& Romero López, D. C Aplicación de las medidas de bioseguridad por parte del profesional de enfermería en la atención integral en el servicio de centro obstétrico del Hospital Provincial General Docente Riobamba periodo octubre 2015 - febrero 2016. Universidad Central del Ecuador. Recuperado de http://www.dspace.uce.edu.ec/handle/25000/7542

20. Rojas Noel, E. E. 2015; Nivel de conocimiento y grado de cumplimiento de las medidas de bioseguridad en el uso de la protección personal aplicados por el personal de enfermería que labora en la estrategia nacional de control y prevención de la tuberculosis de una red de salud - . Repositorio de Tesis - UNMSM. Disponible en : http://cybertesis.unmsm.edu.pe/bitstream/handle/cybertesis/4173/Roj as_ne.pdf?sequence $=1$ \&isAllowed=y

21. S, R. G. Hospitalizacion de niños y adolescentes. Rev. Med Clin. Condes. 2015.16(4):236-277. Recuperado de http://www.clcmovil.cl/Dev_CLC/media/Imagenes/PDF revista médica/2005/4 oct/HospitalizacionDeNinosYAdolescentes-5.pdf

22. Salvando vidas: lavado de manos. 2018; Disponible en: https://www.medigraphic.com/pdfs/medcri/ti-2019/ti193a.pdf

23. Herrera López José Luis, Moyano López Ximena Alejandra Velasco Acurio Evelin Fernanda, Analuisa Jiménez Eulalia Isabel, Mejía Rubio Andrea del Rocío. Conocimientos del personal del área de quirófano en el Hospital General Docente Ambato sobre sepsis quirúrgica. Enfermería Investiga. 2020; 5(2):42-47. Disponible en: <https://revistas.uta.edu.ec/erevista/index.php/enfi/article/view/876>

24. Tamariz Chavarria, F. D. Nivel de conocimiento y práctica de medidas de bioseguridad: Hospital San José, 2016. Horizonte Médico. 2018;18(4):42-49. https://doi.org/10.24265/horizmed.2018.v18n4.06

25. Toribio Felipe, R. Higiene de manos en los centros sanitarios. Junta de Extremadura: Consejería de Sanidad y Dependencia. $2015 \quad$ Recuperado de https://www.seguridaddelpaciente.es/resources/documentos/Higiene Manos/Extremadura/hm_centrossanitarios_doc_directivos.pdf 
26. Vásconez, N., \& Molina, S.Manual de normas de bioseguridad para la red de servicios de salud en el Ecuador. 2010 Disponible en: https://aplicaciones.msp.gob.ec/salud/archivosdigitales/documentosDi recciones/dnn/archivos/LIBRO DESECHOS FINAL.pdf

27. Vélez, C. Conocimientos, actitudes y prácticas de normas y manejo de desechos hospitalarios en el personal de enfermería que labora en el Hospital San Luis de Otavalo, Período Marzo - Agosto 2017. Universidad Técnica del Norte. Disponible en: http://repositorio.utn.edu.ec/bitstream/123456789/3619/1/06 ENF 306 TESIS.pdf

28. Vera, D., Castellanos, E., Rodríguez, P., \& Mederos, T. Efectividad de Guía de Buenas Prácticas en la bioseguridad hospitalaria. Revista Cubana de Enfermeria.2017,33(1):40-51. Disponible

http://scielo.sld.cu/scielo.php?script=sci_arttext\&pid=S0864-

03192017000100006

29. Vera, D., Castellanos, E., Rodríguez, P., \& Mederos, T. 2017; Efectividad de Guía de Buenas Prácticas en la bioseguridad hospitalaria. Revista Cubana de Enfermeria.2017;33(1):40-51. Disponible

http://revenfermeria.sld.cu/index.php/enf/article/view/1208

30. Villegas-Arenas, O. A., Gómez, J., Uriel-López, J., Román R. N., Villa, J. E., Botero, J., \& García, N. Medición de la adherencia a lavado de manos, según los cinco momentos de la OMS. Duazary. 2017; 14(2):169. https://doi.org/10.21676/2389783x.1967

31. Zamudio-Lugo, I., Meza-Chávez, A., Martínez-Sánchez, Y. Miranda-Novales, M. G., Espinosa-Vital, J. G., \& Rodríguez-Sing, R.Estudio multimodal de higiene de manos en un hospital pediátrico de tercer nivel. Boletin Medico del Hospital Infantil de Mexico. 2012;69(5):384-390. 